\title{
A Control Mechanism Based on Virtual IP Camera in Hyper- Connected IoT Networks
}

\author{
Israr Ullah ${ }^{1}$ and Do Hyeun Kim ${ }^{2 *}$ \\ ${ }^{1,2}$ Computer Engineering Department, \\ Jeju National University, Republic of South Korea \\ israrullahkk@yahoo.com, ${ }^{2}$ kimdh@jejunu.ac.kr
}

\begin{abstract}
In the recent past, IoT have received remarkable research attention on many fronts. IoT based applications are designed for health, education, energy, industry, transport, security etc. Many IoT projects and research contributions are geared towards development of prototypes, standards and process automation. We have proposed a system to address scalability and management issues in future IoT world with anticipated growth and hyper connectivity. This work is demonstration of accessing and controlling IP Camera via virtual object using proposed management system. Same approach can be extended for other sensors and actuators in hyper-connected IoT environment to facilitate management tasks. Using this system, we can easily locate a desired device and perform supported operations.
\end{abstract}

Keywords: IoT, Virtual Objects, Hyper connected, Access and Control Management

\section{Introduction}

Applications based on Internet of Things (IoT) are growing rapidly in every walk of life e.g. health, industry, transport, security etc. [1]. Advancement in small chip based device manufacturing has paved the ways for realizing IoT vision. We can have tiny devices, capable of communication, attached ot our daily life objects. Through these devices, we can collect the data about any desired phenomenon, access the device remotely to see its current status and also send control command to alter its status if needed. IoT is a kind of extension of traditional sensor networks (where communication was from sensors to sink only) by adding control component. Concept of smart environment, smart cities and smart homes is based on Internet of things idea. Artificial Intelligent based algorithm can be integrated with IoT to take this idea to next level where devices can autonomously respond to its surrounding environment without requiring human intervention.

Normally, IoT based applications require virtualization of real devices in the form of Virtual Objects (VOs). Later these VOs are used to compose various services and provide access to the physical devices. Several systems can be found in existing literature providing device virtualization in its own way [2]. Complex applications can be built on top of these virtual and service objects as per user needs.

Billions of devices are expected to be connected to Internet in near future through IoT based applications in many sectors. When too many devices will be connected and such a system grows to an extent we call hyper-connected then management of the devices and service will become a challenging task. We have proposed an IoT based fault and configuration management system to address this issue. We display the real devices with an icon by their virtual objects over map with their actual location. User can access and

*Corresponding Author 
control respective devices using its virtual object. In this paper, we demonstrate access and control mechanism for IP camera using its virtual object.

Rest of the paper is organized as follows: Section 2 describe related work. Proposed system architecture is presented in Section 3. System implementation and working is covered in Section 4. Finally, we conclude the paper with an outlook to our future work.

\section{Related Works}

Numerous applications of IoT are geared towards the concept of building smart environment in home, cities and industry. Philips Company have manufactured special bulb under Hue project that can be accessed and controlled (ON/OFF) remotely [3]. Similarly, to build intelligent IoT applications using sematic technologies, a project IoT.est was initiated to support interoperability [4]. Device virtualization through virtual objects and application of semantic ontology for their interacting has been presented in [5-7].

In IoT based networks, service discovery and composition is also a challenging area. Authors in [8] have provide a mechanism for on-demand service creation from existing service in order to fulfill complex user demands using visual and interactive interface. In [9], the authors present a semi-automatic mechanism for service filtering using semantic ontology. Web of Objects (WoO) or Web of Things (WoT) are similar concepts in which web interface is normally used for management and operation. To achieve energy efficiency in smart homes, authors in [10] have presented a model for service composition using semantic ontology in WoO. For efficient utilization of energy in smart homes, a WoO based system is developed in [11] semantic ontologies. Services are not only composed of virtual objects for physical devices but also application can be used a service in IoT environment. "Buddy Thing" is an idea presented in [12] in order to provide browsing as a service in IoT.

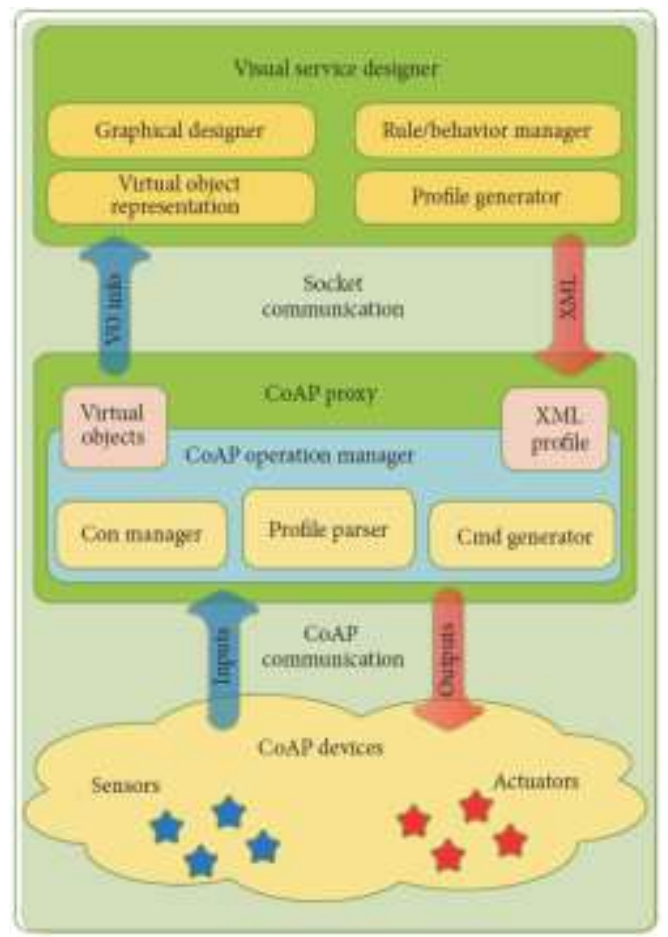

Figure 1. Conceptual Architecture for the DIY Visual Service Design [16]

Artificial Intelligent based algorithm can be integrated with IoT to take this idea to next level where devices can autonomously respond to its surrounding environment without 
requiring human intervention. Using AI techniques, context-aware services are composed using context data collected from IoT sensors in order to build intelligent systems. Intelligent context-aware services based applications have been discussed in [13-15].

Do-it-Yourself (DIY) based projects have attracted tremendous research attention. [16] presents an improved DIY based service composition toolbox using CoAP protocol as shown in Figure 1. They have developed a visual interface for composition of services using registered virtual objects using drag and drop operations. Arduino [17], Raspberry Pi [18] and Intel Edison Board [19] boards are introduced to make it easy to build IoT based test applications for normal users. SAM project by Kickstarter [20] company is also very popular tool for learners.

To enable smart communication among IoT devices, constrained application protocol (CoAP) is developed by IETF which is light weight protocol with support for HTTP protocol for internet access [21].

Many IoT projects and research contributions are geared towards development of prototypes, standards and process automation. We have proposed a system to address scalability and management issues in future IoT world with anticipated growth and hyper connectivity. This work is demonstration of accessing and controlling IP Camera via virtual object using proposed management system.

\section{Proposed System Architecture}

Our proposed system conceptual architecture is given in Figure 2. Through device virtualization process, we transform real device properties and stored it in a database in the form of virtual objects. Virtual objects hold real devices properties and try to capture it behavior through supported control operations. Afterwards, we use fault management to identify and isolate any faulty device in the system for repair or replacement. Configuration management provides an interface to user to interact with actual devices to perform supported control operations.

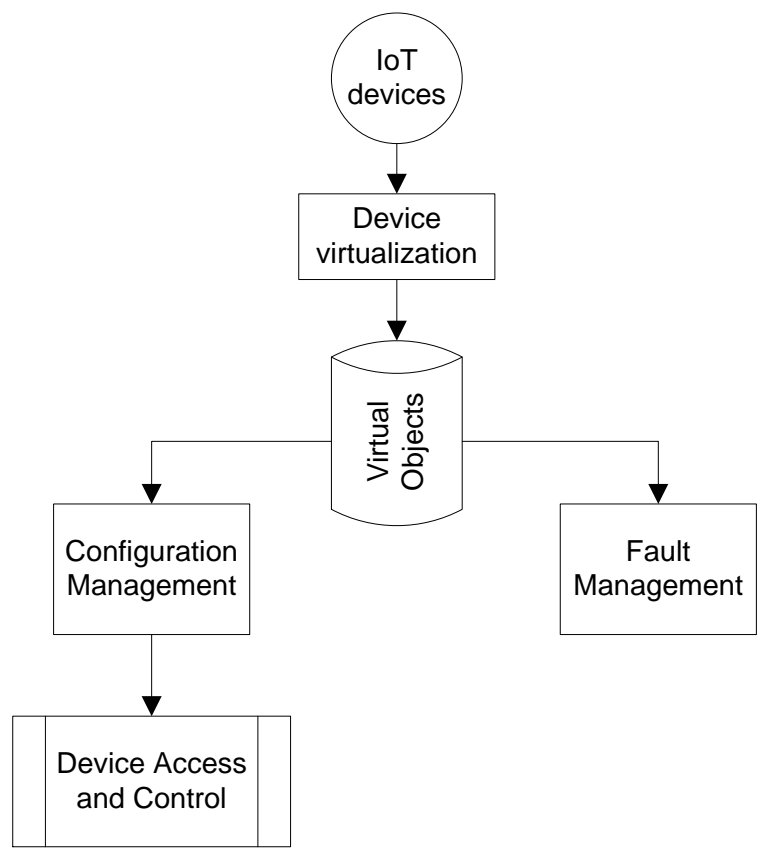

Figure 2. Conceptual Architecture of Proposed System

Detailed working of fault management is shown in Figure 3. We can either check device current working status or fault status. Through working status, we get to know if selected device is currently being used by any other service or free. Preferably, idle 
devices shall be used in newly composed services for load balancing and improve overall response time. If a single device is being used by many services then it will certainly become bottleneck point and degrade overall system response which may be crucial in delay sensitive applications. Using device fault system, we can identify faulty device that are not responding due to some unknown reasons. The device may have been damaged or there may be some network connectivity issue. Anyhow, this will allow easy identification of faulty devices for repair or replacement.

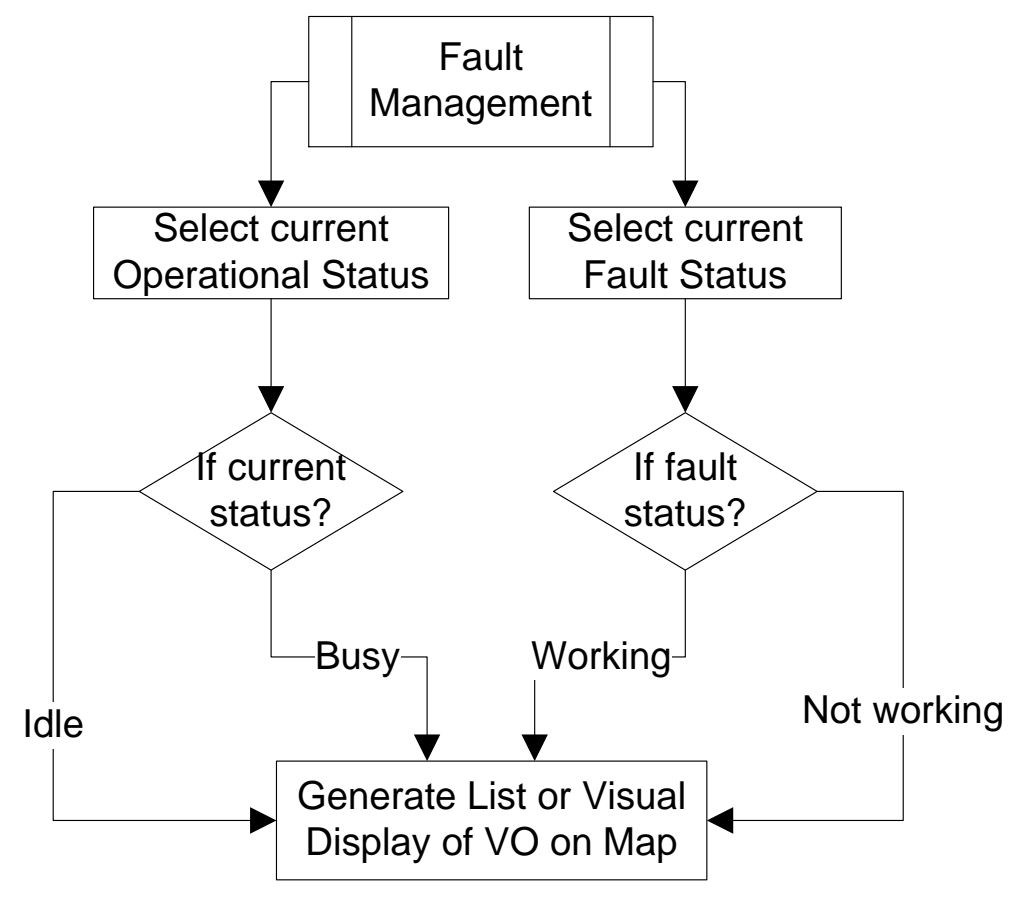

Figure 3. Fault Management to Identify Device Current and Fault Status

Detailed working of configuration management is shown in Figure 4. We can have configuration management in three different ways (a) Location based: here user just selects a particular location over map where he/she want to find some device. The system will automatically list available device within the range of user selected location. User can specify and adjust the range as per choice. (b) Device type based: here user can choose his/her required device type and the system will list all available devices of selected type. (c) Hybrid: This is combination of earlier two approaches i.e. user can specify required device type and location. This will help easy finding of a particular device type within a given location. 


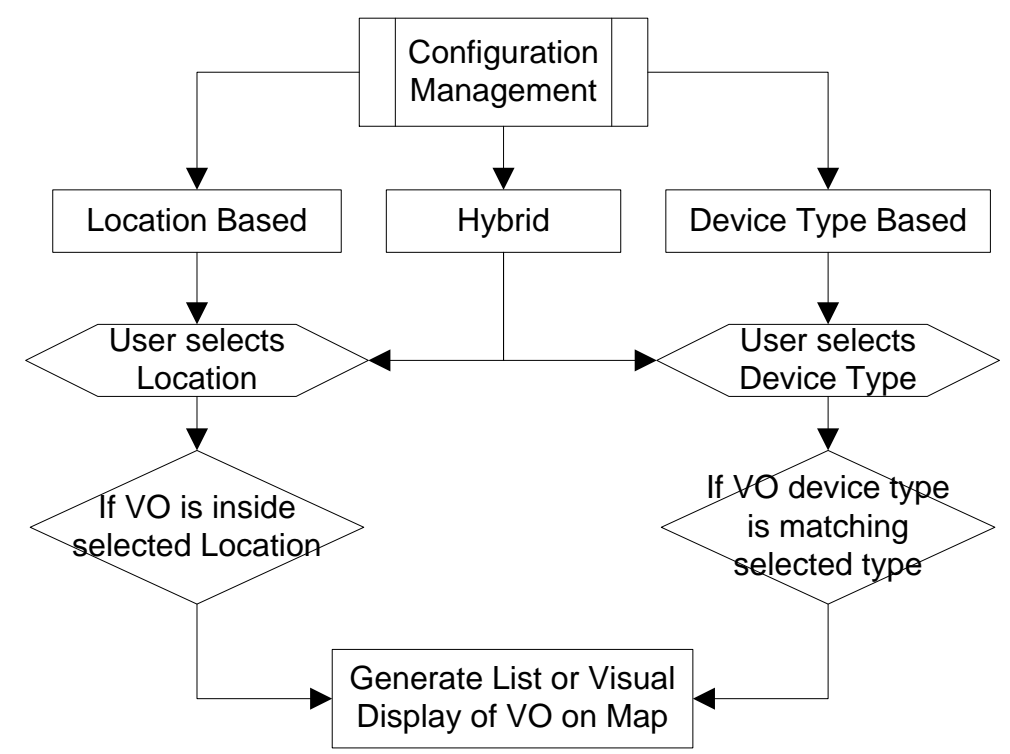

Figure 4. Configuration Management for Device Access and Control

For access and control, user can select any device to perform supported interactive operations through configuration management. As shown in Figure 5, user will select the desired device. Afterwards, current operation status of the device is checked if not working then appropriate error message is displayed. If device is working properly then application use the device profile information and build a user interface to allow users to perform supported interactive operations. User commands are sent to the respective device using device URL.

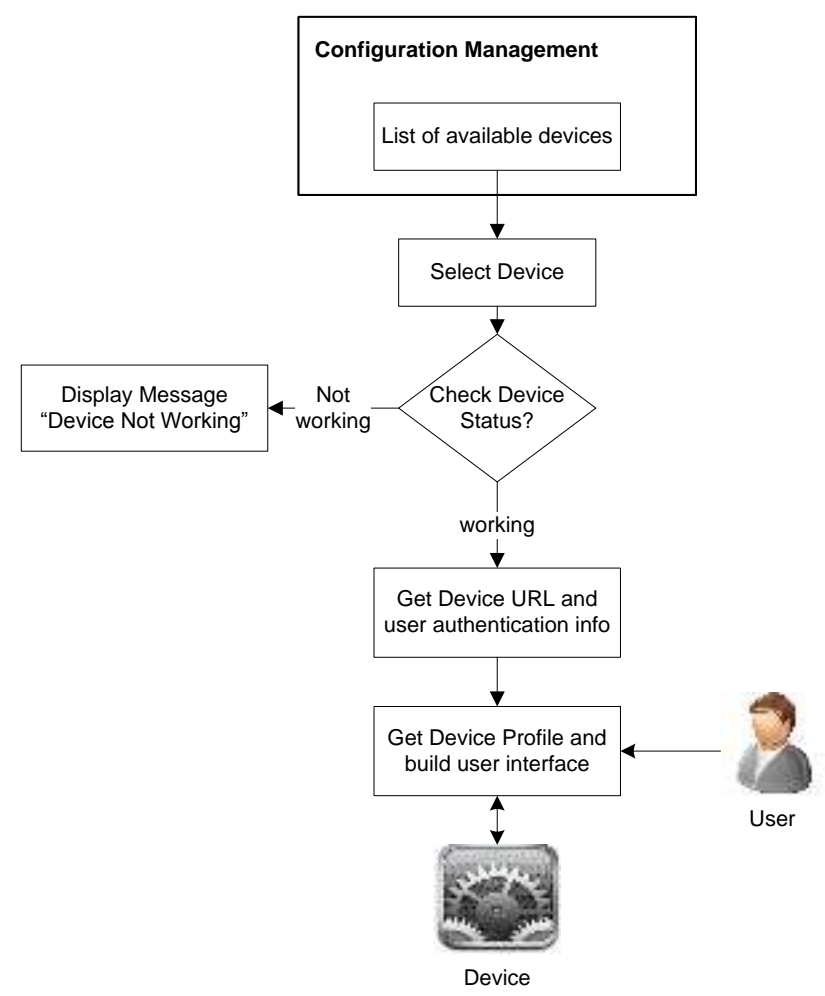

Figure 5. Device Access and Control Mechanism Using Configuration Management 
Using virtual object configuration management, user can select any device to perform supported interactive operations. Here, we present interactive operations for IP Camera only as shown in Figure 6. After selecting the camera, first its current operational status will be checked. If "not working" then appropriate message will be displayed to user. Otherwise camera profile information will be used to create live stream from camera. Camera control operations can also be performed if supported by camera API.

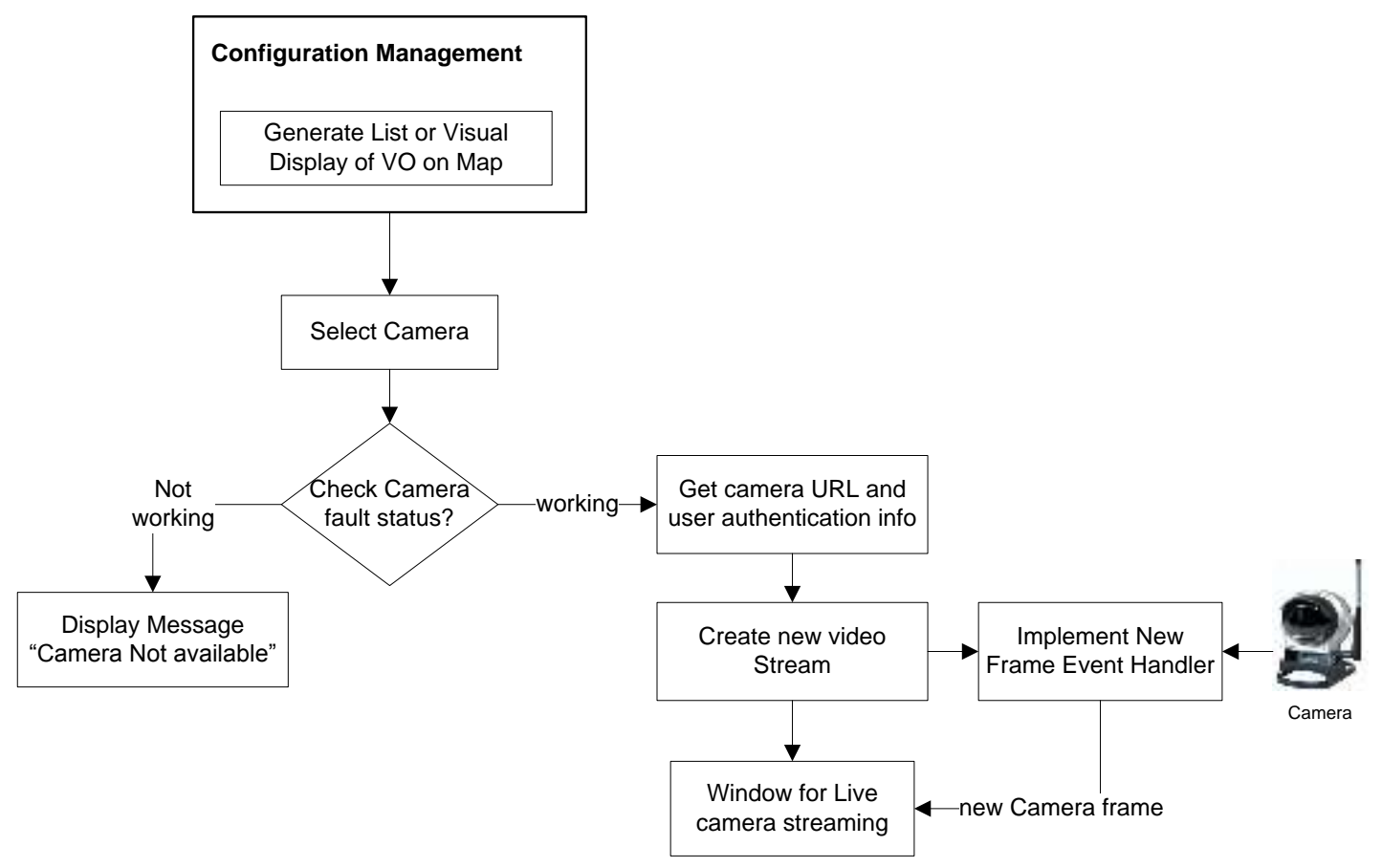

Figure 6. Mechanism for IP Camera Access and Control

In VOMS, when IP camera is selected then pop window is opened for camera view. After establishing connection with the Camera, frame queue is created to hold frames from streaming object. Streaming object runs in separate loop to get continuous frames from camera as per frame rate which are then stored in frame queue. If we want to save the stream then Camera thread is initiated to get the frames from queue and store it. This sequence of operations is illustrated in Figure 7.

\section{Implementation}

We have implemented a prototype application for management of IoT services and virtual objects in Visual Studio 2015 using C\#. Microsoft SQL Server is used to store profile information of registered IoT services and virtual objects.

In order to have virtual object for IP Camera, we create its profile in Virtual Device Manager application as shown in Figure 8. Typical virtual object profile for the camera has information as shown in xml format in Figure 9. 


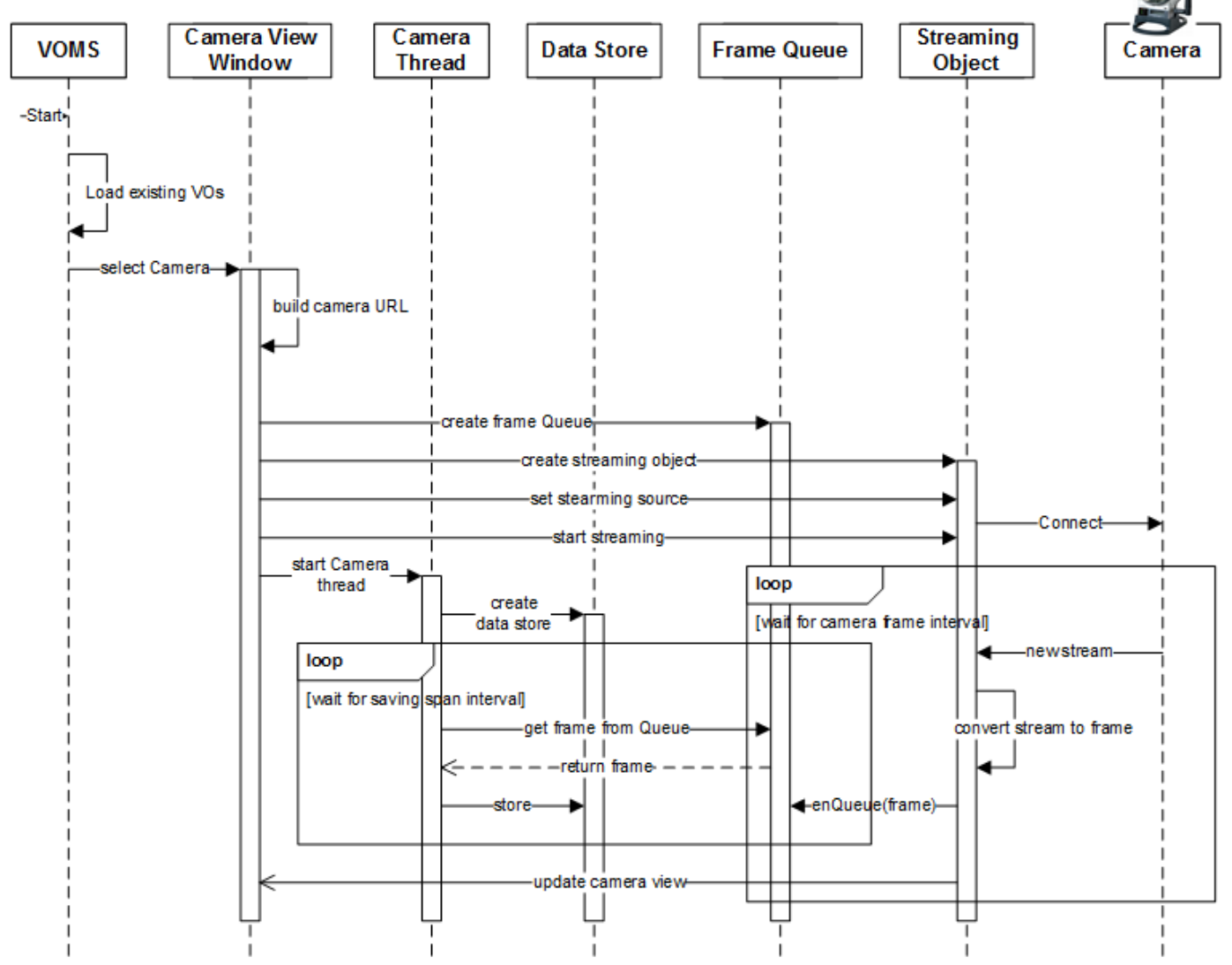

Figure 7. Sequence Diagram of Proposed System for IP Camera Streaming and Storage

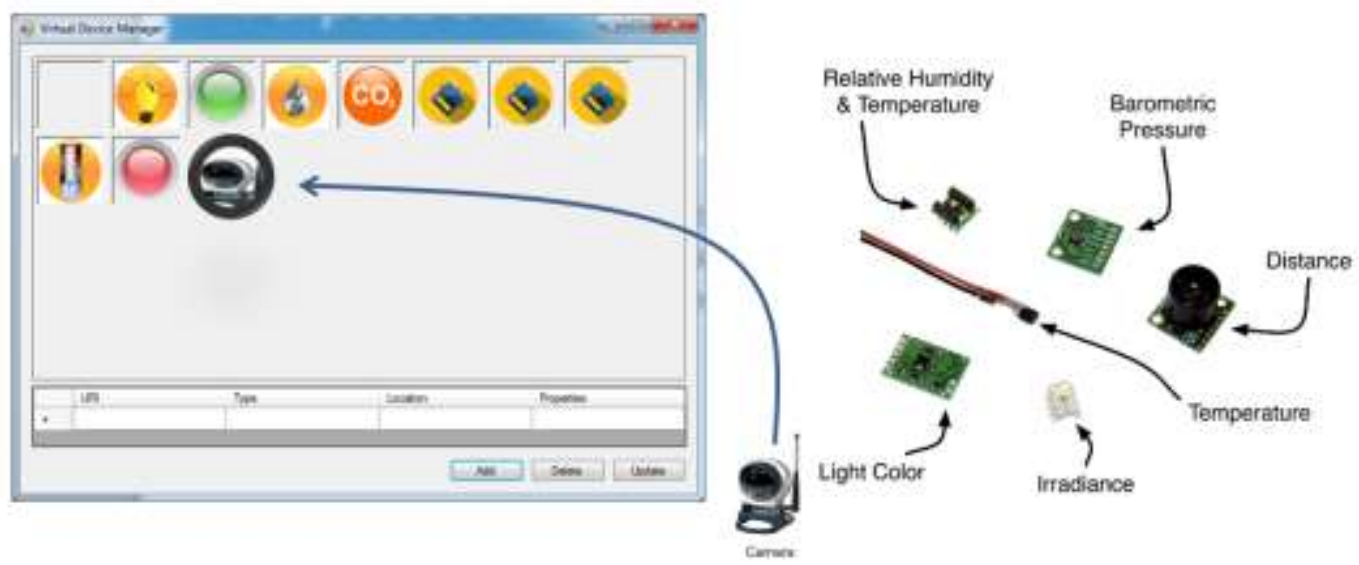

Figure 8. Creating Virtual Object for IP Camera Using Virtual Device Manager 


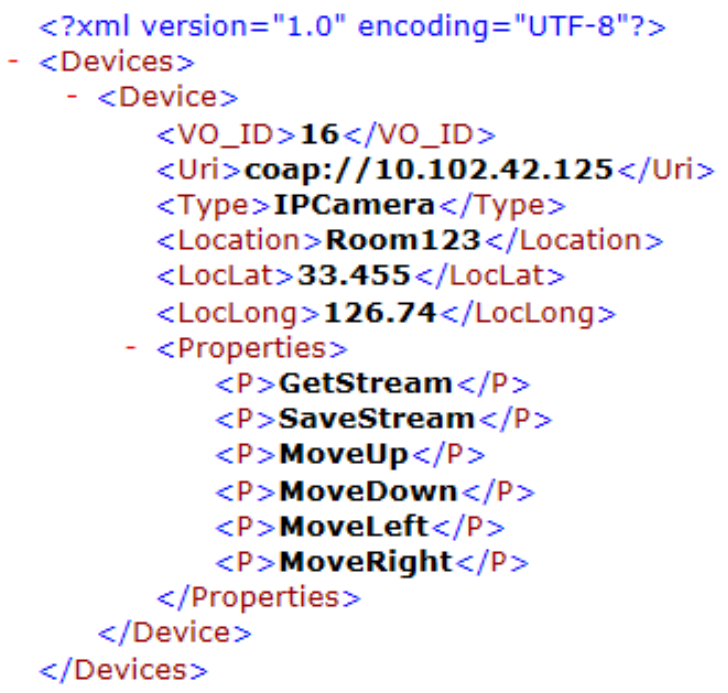

Figure 9. Typical IP Camera Profile (xml view)

Camera profile holds information about camera properties including its URI and location information. Properties sections hold supported operations for the designated camera. We can get stream from camera for live view and can save the stream in a video file for later retrieval and analysis. Furthermore, various control operations are also included to move the camera in four directions to get desired view.

Database of schema of our implementation is shown in Figure 10. We have four main tables to hold information of devices properties in virtual object format. These information can be used to retrieve the available devices profiles for later updation and necessary modifications if needed. Furthermore, we can retrieve devices profiles stored in the database and export it in xml format to be used by any other application or share it with others.

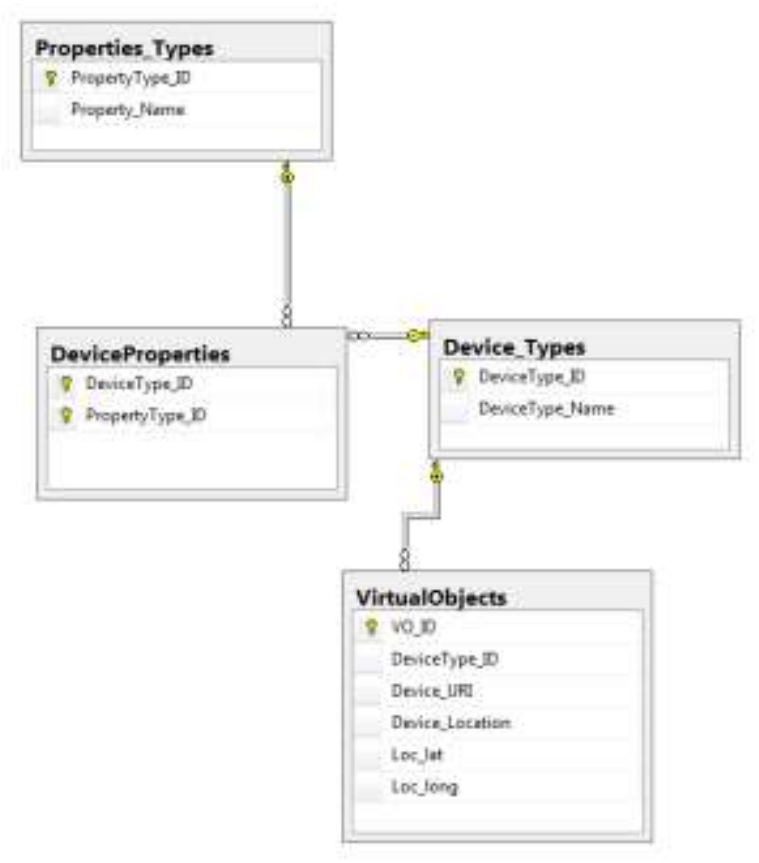

Figure 10. Database Schema 
Next, we use this virtual objects information in Virtual Object Management System application to remote access and control of IP Camera along with other sensors and actuators as shown in Figure 11.

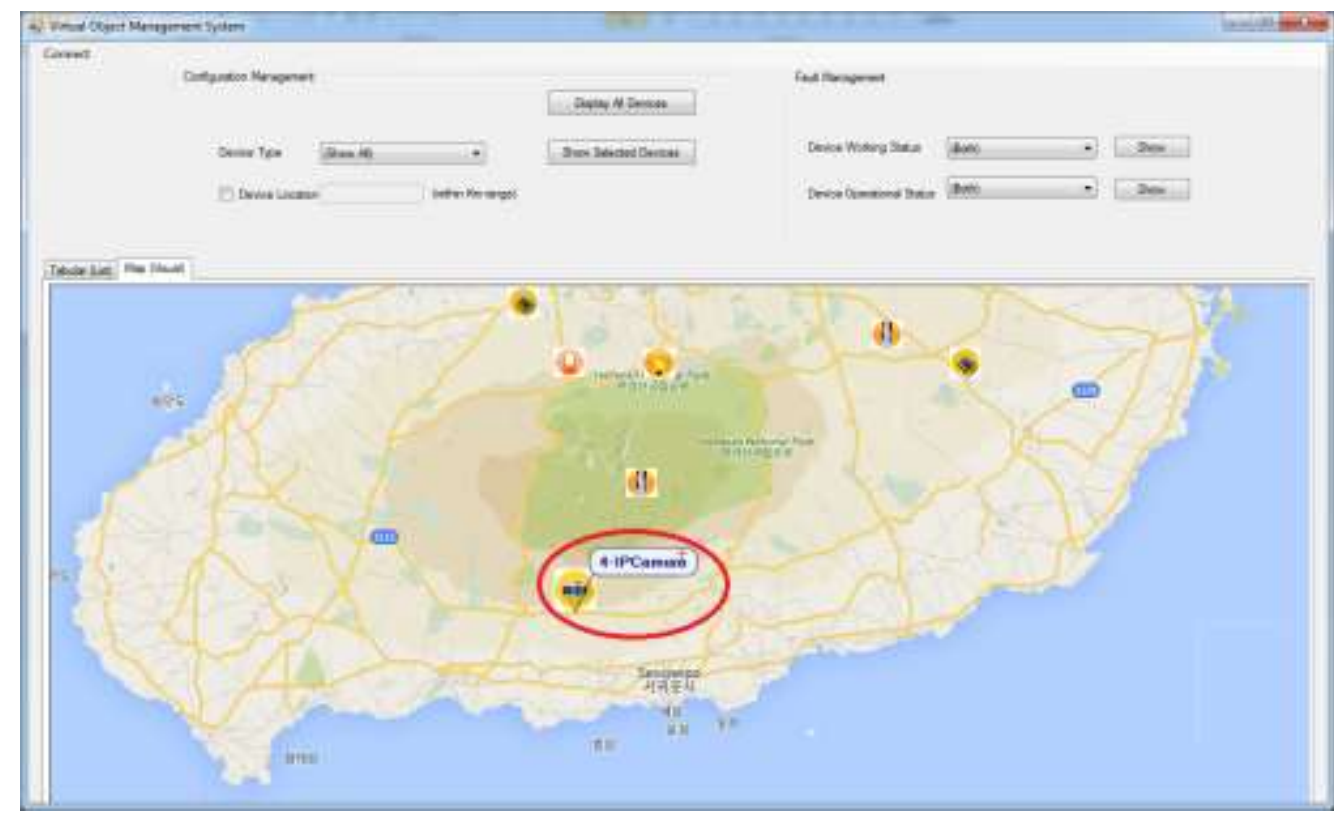

Figure 11. Select the IP Camera to Get Its Streaming View in a Pop-Up Window

Streaming view from selected IP camera is shown in pop-window shown in Figure 12. We can perform various supported control operations e.g. move the camera in four directions. Stream from the camera can be saved in some online storage for later retrieval and analysis.

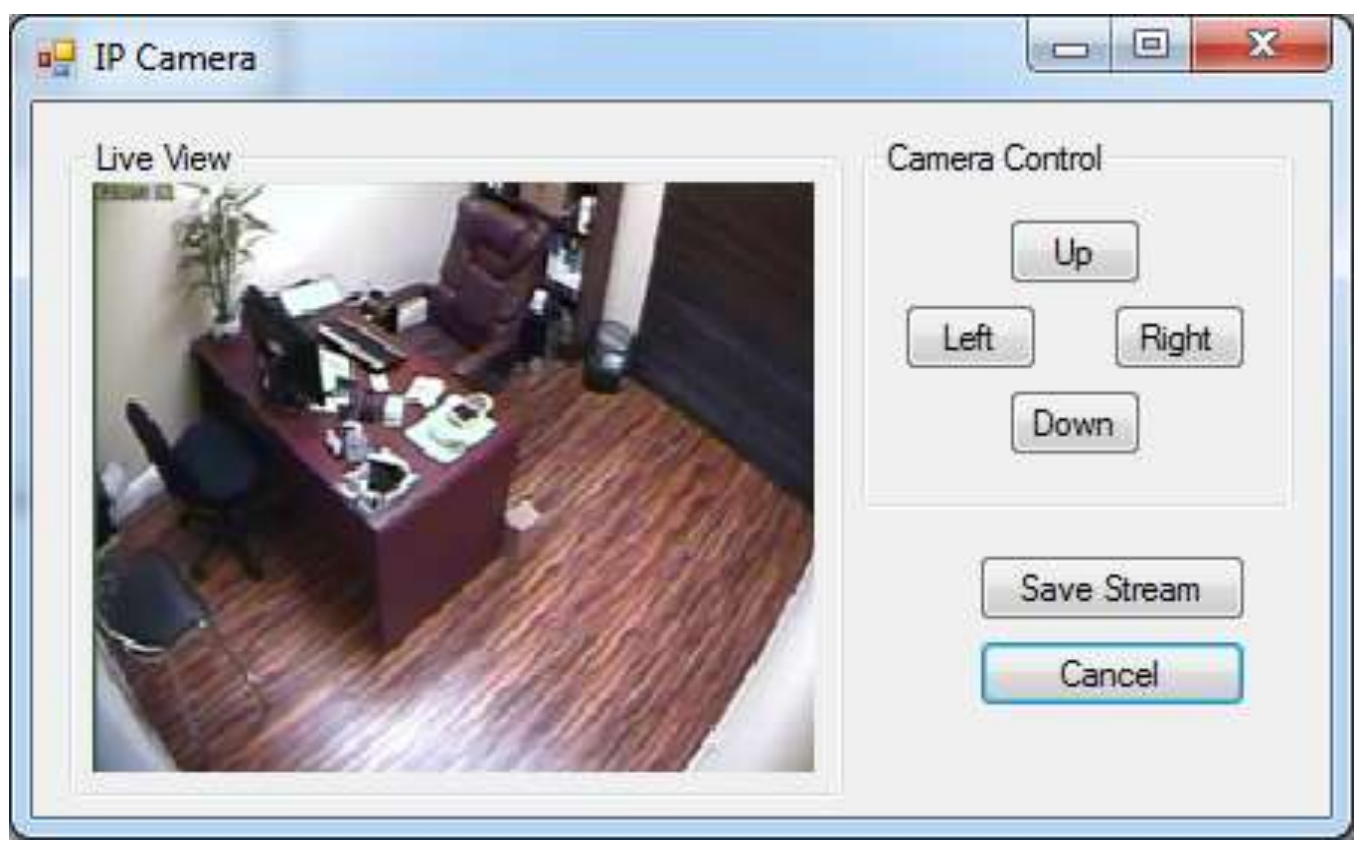

Figure 12. Popup Window for Live IP Camera Streaming 


\section{Conclusions}

This work demonstrates accessing and controlling of IP camera using its virtual object profile. Same approach can be extended for all other sensors and actuators in any hyperconnected IoT environment to facilitate management tasks. Using this system, we can easily locate a desired device and perform supported operations. This work is demonstration of accessing and controlling of IP camera via its virtual object. In future, we will try to extend this system by integrating it with composition tool to facilitate IoT based application development. With the help of such system, any user can build his/her desired IoT based application without requiring sound technical skills.

\section{Acknowledgment}

This research was supported by the 2016 scientific promotion program funded by Jeju National University. Correspondence related to this paper should be addressed to DoHyeun Kim; kimdh@jejunu.ac.kr.

\section{References}

[1] D. Miorandi, S. Sicari, F. D. Pellegrini, I. Chlamtac, "Internet of things: Vision, applications and research challenges", Ad Hoc Networks, vol. 10, no. 7, (2012), pp. 1497 - 1516.

[2] D. Mazzei, G. Fantoni, G. Montelisciani and G. Baldi, "Internet of Things for designing smart objects," 2014 IEEE World Forum on Internet of Things (WF-IoT), Seoul, (2014), pp. 293-297.

[3] Philips Hue, http://www.developers.meethue.com/, [Online; accessed 12-Oct-2016] (2016).

[4] De Suparna, Carrez, Test-Enabled Architecture for IoT Service Creation and Provisioning, Springer Berlin Heidelberg, Berlin, Heidelberg, (2013), pp. 233-245.

[5] N. Yamada, K. Sakamoto, G. Kunito, Y. Isoda, K. Yamazaki, and S. Tanaka, "Applying Ontology and Probabilistic Model to Human Activity Recognition from Surrounding Things," IPSJ Digital Courier, vol. 3, (2007), pp. 506-517.

[6] Do-it-Yourself Smart Experiences (DiY Smart Experiences). D3.1: Service Ontologies, ITE2 project 08005. Available online: https://itea3.org/project/diy-smart-experiences.html (accessed on 13 February 2015).

[7] Xiao Hang Wang, Da Qing Zhang, Tao Gu and Hung Keng Pung, "Ontology Based Context Modeling and Reasoning using OWL", In proceedings of the Second IEEE Annual Conference on Pervasive Computing and Communications Workshops (PERCOMW'04), (2004); Orlando, FL, USA.

[8] M. Naeem, R. Heckel, F. Orejas, Semi-automated service composition using visual contracts, in: Proceedings of the 7th International Conference on Frontiers of Information Technology, FIT '09, ACM, (2009); New York, USA.

[9] E. Sirin, J. Hendler, B. Parsia, Semi-automatic composition of web services using semantic descriptions, in: InWeb Services: Modeling, Architecture and Infrastructure workshop in ICEIS 2003, (2002), pp. 1724.

[10] S. S. Ara, Z. U. Shamszaman, and Ilyoung Chong, "Web-of-Objects Based User-Centric Semantic Service Composition Methodology in the Internet of Things", International Journal of Distributed Sensor Networks, Vol. 2014, Article ID 482873

[11] M. G. Kibria and I. Chong, "A WoO based knowledge driven approach for smart home energy efficiency," 2014 International Conference on Information and Communication Technology Convergence (ICTC), Busan, 2014, pp. 45-50.

[12] N. K. Giang, M. Ha and D. Kim, "Buddy Thing: Browsing as a Service for the Internet of Things," 2015 IEEE International Conference on Services Computing, New York, NY, 2015, pp. 122-129.

[13] BUTLER Project. Available online: http://www.iot-butler.eu (accessed on 21 April 2015).

[14] W. Yao and A. Kumar, "CONFlexFlow: Integrating flexible clinical pathways into clinical decision support systems using context and rules”, Decision Support System, 2013, 55, 499-515.

[15] Y. Liu, Boon-Chong Seet, and Adnan Al-Anbuky, "An Ontology-Based Context Model for Wireless Sensor Network (WSN) Management in the Internet of Things", Journal of Sensor and Actuator Network, 2015, 2, 653-674.

[16] M. S. Khan, D. Kim, DiY interface for enhanced service customization of remote IoT devices: A coap based prototype, International Journal Distributed Sensor Network. 2015 (2016) 185:185-185:185.

[17] Arduino, https://www.arduino.cc/, [Online; accessed 05-Oct-2016] (2016).

[18] R. Pi, https://www.raspberrypi.org/, [Online; accessed 05-Oct-2016] (2016).

[19] Intel Edison Board, https://software.intel.com/en-us/iot/hardware/edison, [Online; accessed 13-Oct2016] (2016). 
[20] SAM: The Ultimate Internet Connected Electronics Kit, https://www.kickstarter.com , [Online; accessed 08-Oct-2016] (2016).

[21] A C\# implementation of the CoAP protocol, https://github.com/smeshlink/CoAP.NET, [Online; accessed 08-September-2016] (2016).

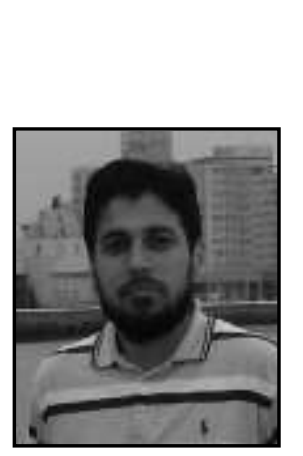

\begin{abstract}
Authors
Israr Ullah, received his MCS degree from Institute of Computing and Information Technology (ICIT), Gomal University, Pakistan, in 2004. He completed his M.S. in computer science from National University of Computer and Emerging Sciences (NUCES), Islamabad, Pakistan, in 2009. Currently, he is pursuing his Ph.D. studies at Computer Engineering Department, Jeju National University, Republic of Korea. His research work is focused on application of prediction and optimization algorithms to build IoT based solutions. His research interests also include analytical modeling, network simulation and analysis of optimization algorithms.
\end{abstract}

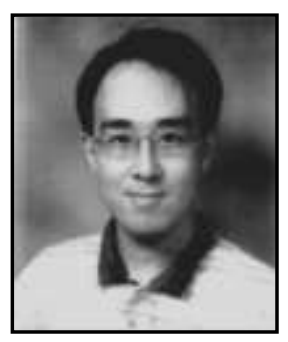

Do-Hyeun Kim, He received the B.S. degree in electronics engineering from the Kyungpook National University, Korea, in 1988, and the M.S. and Ph.D. degrees in information telecommunication the Kyungpook National University, Korea, in 1990 and 2000, respectively. He joined the Agency of Defense Development (ADD), from Match 1990 to April 1995. Since 2004, he has been with the Jeju National University, Korea, where he is currently a Professor of Department of Computer Engineering. From 2008 to 2009, he has been at the Queensland University of Technology, Australia, as a visiting researcher. His research interests include sensor networks, M2M/IOT, energy optimization and prediction, intelligent service, and mobile computing. 
International Journal of Control and Automation

Vol. 10, No. 5 (2017) 